\title{
Distribution D'une Nouvelle Adventice, Porophyllum Ruderale (Asteraceae), Des Cultures De Côte d'Ivoire
}

\author{
Kpla A Christine Florence, Doctorante \\ Touré Awa, Maître-assistante \\ Gué Arsène, Docteur \\ Ipou Ipou Joseph, Maître de Conférences
}

Université Félix Houphouët Boigny, UFR Biosciences, Côte d'Ivoire

Doi: 10.19044/esj.2018.v14n36p333 URL:http://dx.doi.org/10.19044/esj.2018.v14n36p333

\begin{abstract}
The presence of Porophyllum ruderale is increasingly recognized in different agroecosystems in Côte d'Ivoire. The current study is initiated to determine its distribution area and abundance. To achieve our goals, data were collected through surveys of farmers and field sampling was conducted to assess the abundance of Porophyllum ruderale. These were processed by the Correspondence Analysis (AC), and with a GPS, geographic coordinates were incorporated into the ArcGIS software to identify colonized areas. Forest regions (Adzopé and Daloa) are the most infested with a centesimal frequency (Fr) of $100 \%$ of Porophyllum ruderale. In the other localities (Yamoussoukro, Man, Bondoukou) the frequencies vary from 43 to $93 \%$. This species is rare in the Korhogo region with a zero centesimal frequency. Agroecological zones favorable to the development of the weed have a rainfall amounting from 1100 to $2400 \mathrm{~mm}$ and a temperature between 21$28 \mathrm{C}$. Of the various agrosystems encountered, cassava is the crop with the highest presence and the highest density of Porophyllum ruderale with more than 21 individuals $/ \mathrm{m}^{2}$. At the end of this study, Porophyllum ruderale is present in most of Ivorian territory, particularly in forest areas where agriculture is the main activity.
\end{abstract}

Keywords: Weed, Distribution, Porophyllum ruderale, Côte d'Ivoire

\section{Resume}

La présence de Porophyllum ruderale est de plus en plus constatée dans les différents agroécosystèmes de Côte d'Ivoire. Cette étude est initiée afin de déterminer son aire de répartition et son abondance. Pour atteindre nos objectifs, les données ont été collectées à travers des enquêtes auprès des agriculteurs et des échantillonnages ont été réalisés dans les champs pour 
évaluer l'abondance de Porophyllum ruderale. Les données recueillies ont été traitées par l'Analyse Factorielle des Correspondances (AFC), puis grâce à un GPS, les coordonnées géographiques ont été incorporées au logiciel ArcGIS pour identifier les zones colonisées. Les régions forestières (Adzopé et Daloa) sont les plus affectées avec une fréquence centésimale (Fr) de $100 \%$ de présence de Porophyllum ruderale. Dans les autres localités (Yamoussoukro, Man, Bondoukou), les fréquences varient de 43 à $93 \%$. Cette espèce est rare dans la région de Korhogo avec une fréquence centésimale nulle. Les zones agroécologiques favorables au développement de l'adventice ont une pluviométrie s'élevant de 1100 à $2400 \mathrm{~mm}$ et une température comprise entre $21-28^{\circ} \mathrm{C}$. Parmi les différents agrosystèmes rencontrés, le manioc est la culture qui présente la plus grande présence et la plus forte densité de Porophyllum ruderale avec plus de 21 individus $/ \mathrm{m}^{2}$. Au terme de cette étude, Porophyllum ruderale est présente dans la majeure partie du territoire ivoirien, particulièrement dans les zones forestières où l'agriculture est la principale activité.

Mots-clés: Mauvaise herbe, distribution, Porophyllum ruderale, cultures, Côte d'Ivoire

\section{Introduction}

Porophyllum ruderale Jacq. Cass., plus connu sous le nom de la "coriandre bolivienne" ou d' "arnica-brésilienne", est une plante originaire d'Amérique tropicale (Fonseca et al., 2006). En Côte d'Ivoire, on observe une soudaine apparition et prolifération de cette adventice sur les parcelles agricoles. Cette plante annuelle, herbacée, à tige ramifiée, se propage principalement par les graines. De la famille des Asteraceae, Porophyllum ruderale produit beaucoup de semences avec un taux de germination élevé. Les travaux de De Marinis et al.(1980) ont obtenu plus de 80\% de levées et ceux de Yao et al. (2017) s'élèvent à 98\%. Dans des milieux naturels (jachères) Kpla et al. (2017) ont estimé un taux variant de 40 à $70 \%$. Le fruit nommé akène, est un fruit sec indéhiscent, très léger, présentant une touffe de poils à l'extrémité (Frangiote-Pallone \& De Souza, 2012). Ces organes annexes, comme ceux de Tridax procumbens (Asteraceae), et de Chromolaena odorata (Asteraceae), facilitent la dispersion sur de longues distances, se collant facilement au pelage des animaux et aux vêtements des hommes ou transportés par le vent (Johnson, 1997). Cette espèce s'adapte à différents types de sols même pauvres ou sableux (Kissmam \& Groth, 1999). L'adventice se rencontre aux bords des routes et pistes, dans les jachères, les décombres, mais surtout dans les espaces ouverts naturellement ou perturbés par l'homme notamment les champs cultivés (Kissmam \& Groth, 1999 ; Frangiote-Pallone \& De Souza, 2014). Porophyllum ruderale fut déjà signalé comme plante 
invasive en Amérique latine par De Marinis et al. (1980) et par Kissmam \& Groth (1999). Cette mauvaise herbe constitue une des principales contraintes qui affecte les rendements des productions agricoles dans la région de la Mé en Côte d'Ivoire (Yapi, 2017). En effet, les mauvaises herbes peuvent entraîner des baisses de rendement évaluées à plus de 2,2 millions de tonnes par an en Afrique sub-saharienne (Rodenburg \& Johnson, 2009). Ces bioagresseurs sont de véritables menaces pour la sécurité alimentaire. Pour définir une meilleure gestion des adventices spécifiquement de Porophyllum ruderale, cette étude est entreprise afin de localiser les zones de colonisation. Il s'agira donc de: -identifier les zones de présence de l'adventice afin d'établir une carte de sa répartition;

-connaître les conditions agroécologiques (pluviométrie et température) favorables à son développement;

-évaluer les paramètres (séquences topographiques et les modes de désherbages et le type de culture) qui pourraient influencer sa répartition et son abondance.

\section{Matériel et méthodes \\ Matériel}

Le matériel biologique est composé de l'adventice $P$. ruderale et des différentes cultures en présence. Le matériel technique est constitué d'un GPS (Global Positioning System) qui a servi à localiser les différents sites et des fiches d'enquêtes.

\section{Zones d'études}

Six sites, choisis sur la base de leur localisation dans des zones agroécologiques bien différenciées (Figure 1) où la production vivrière est importante, ont permis de conduire cette étude. Ils ont concerné spécifiquement les chefs-lieux des régions :

- pour le sud (climat de type équatorial appelé climat Attiéen, zone forestière) : la région de la Mé avec Adzopé comme chef-lieu; avec une pluviométrie annuelle moyenne de $1500 \mathrm{~mm}$ et une température de 21 à $27^{\circ} \mathrm{C}$ (Yapi, 2017);

- pour l'est : la région du Gontougo avec Bondoukou le chef-lieu; (zone de transition entre le climat équatorial et soudanais nommé climat Baouléen atténué, zone forestière et de savane pré-forestière (Youan, 2008). Les températures moyennes sont comprises entre $22-27^{\circ} \mathrm{C}$ et les précipitations moyennes annuelles (mm/an) sont de 1100 à 1700 (Krogba et al., 2016);

- pour le centre-ouest, la région du Haut-Sassandra, Daloa est le chef- lieu; caractérisé par le climat tropical humide de transition, zone forestière, les températures moyennes sont de $26^{\circ} \mathrm{C}$ et les pluies annuelles s'élèvent à 1276 mm (Tra Bi et al., 2015). 
- pour le nord (climat soudanais) : la région du Poro dont Korhogo est le chef-lieu; zone savanicole présentant une température moyenne s'élevant de 24 à $36^{\circ} \mathrm{C}$ (Coulibaly et al., 2014), avec 800 à $1200 \mathrm{~mm}$ de pluies annuelles (Krogba et al., 2016).

- pour l'ouest (zone équatorial caractérisée par un climat de montagne) : la région du Tonkpi, Man est le chef-lieu; zone de forêt dense humide, les températures sont douces avec une moyenne de $24^{\circ} \mathrm{C}$ et une pluviométrie annuelle de 1300 à $2400 \mathrm{~mm}$ (Bakayoko et al., 2014).

- pour le centre (climat de transition entre équatorial et soudanais nommé Baouléen) : le District Autonome de Yamoussoukro où celui-ci est le cheflieu ; les températures moyennes sont comprises entre 22 et $27^{\circ} \mathrm{C}$ et les précipitations moyennes annuelles varient de 1100 à 1700 (Krogba et al., 2016).

\section{Méthodes}

\section{Collecte de données}

\section{Réalisation des enquêtes}

Les rencontres avec les agriculteurs ont été possibles grâce au ministère de l'agriculture (MINAGRI) régional qui a facilité le contact avec des coopératives vivrières. Le choix des villages s'est effectué selon la provenance du producteur et au moins trois villages situés sur des axes routiers différents ont été échantillonnés. Les interviews avec les agriculteurs ont eu lieu au champ où ils répondent aux questionnaires (fiche d'enquête) et une photographie de l'adventice leur est présentée. Les échanges ont permis d'apprécier quelques informations sur $P$. ruderale (connaissance de l'adventice, année d'observation, période d'abondance, utilisation de la plante). Puis, les données concernant le mode de désherbage, le type de culture pratiquée par le producteur ont été notés ainsi que la localité et la topographie de la parcelle. 


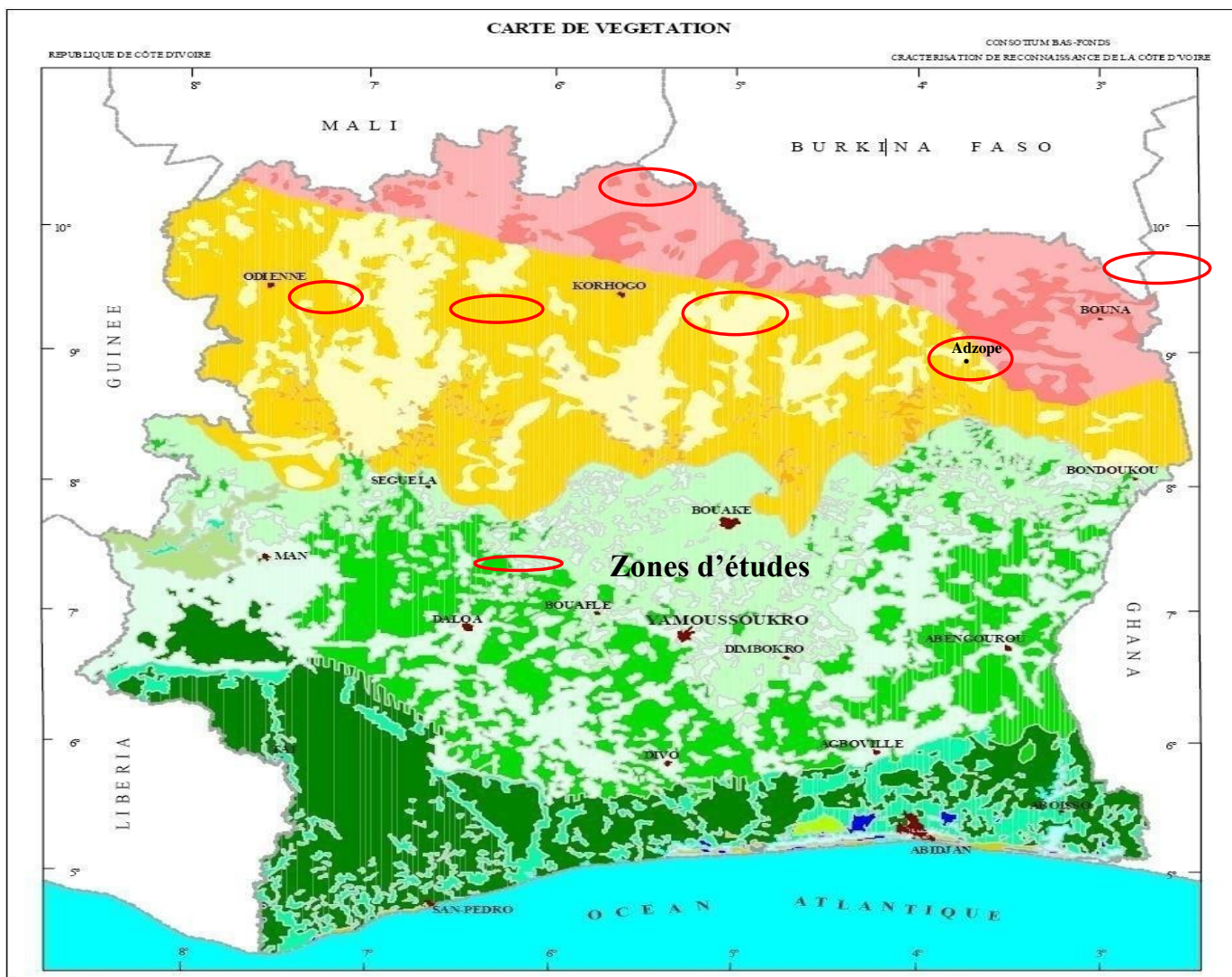

\section{I- DOMAINE GUINEEN}

A- SECTEUR LITT ORAL

Forêt et zone agricole

Savane littorale

Mangrove

B- SECTEUR OMBROPHILE

Forêt dense humide sempervirente

Forêt marécageuse

Forêt défrichée ombrophile

Savane ombrophile

BUREAU NATIONAL DE TUDES TEC HNIQUES ET DE DEVELOPPE MENT CENTRE DE CARTOGR APHIE ET DE TELEDETECTION Forêt et prairies
II- DOMAINE SOUDANAIS

C- SE CTEUR MES OPHUL

Forêt dense humide s emi-décidue Forêt défrichée mésophile Savane mésophile

D. SECTEUR MONTAGNARD A- SECTEUR SUB-SOUDANAIS
Forêt dense sèche Forêt cl aire sèche et/ou savane boisée sub-soudanaise - Savane arborée et/ou savane arbustive sub-soudanaise

B-SECTE UR SOUDANAIS

Forêt $\mathrm{cl}$ aire sèche et/ou Forêt cl aire sèche et/ou
savane boisée soudanaise

Savane arborée et/ou arbustive soudanaise

SOURCE : Atlas de Côte d'Tvoir e, ORSTOM-IGT, 1979. carte à 1/2 000 000. Rédigée par rUTSIG, août 1999.

Figure 1:Végétation de la Côte d'Ivoire et localisation des zones d'études (Source: BNETD/CCT, 2007)

L'interview terminée, une recherche de $P$. ruderale est entreprise sur la parcelle. L'aire d'échantillonnage est une surface de $2500 \mathrm{~m}^{2}$ soit $1 / 4$ d'hectare qui offre une bonne homogénéité et considérée comme une unité relativement homogène (Le Bourgeois, 1993). Une cinquantaine de parcelles sont échantillonnées par chef-lieu de région afin d'estimer la densité et la fréquence de $P$. ruderale. Tous les différents types d'agrosystèmes (cultures 
vivrières, pérennes, maraîchères) et quelques jachères ont été considérés mais l'accent est mis sur les cultures vivrières. Celles-ci permettent l'émergence des mauvaises herbes à cause de leur structure aérée. Les cultures arboricoles (café, cacao, hévéa, anacarde) limitent la colonisation des adventices dû à l'ombrage qu'elles créent. Quant aux cultures maraîchères, elles sont pratiquées sur de très petites surfaces où l'entretien est minutieux. La présence ou l'absence de $P$. ruderale (fréquence) est notée sur les fiches d'enquêtes et les coordonnées géographiques des champs sont enregistrées à l'aide du GPS. Lorsqu'elle est présente alors sa densité (individus $/ \mathrm{m}^{2}$ ) est estimée. En outre, la présence d'un individu adulte de l'adventice dans d'autres villes (que nous avons visitées) en dehors de nos sites d'études, observée aux abords des routes ou dans des exploitations agricoles a été prise en compte. Toutes ces informations recueillies ont permis d'établir l'aire de répartition de $P$. ruderale. Le nombre de parcelles échantillonnées est de 328 et 291 agriculteurs ont renseigné nos fiches.

\section{Exploitation des données}

La codification de Bayer (1992) à six lettres est utilisée pour les variables excédent ce chiffre. Pour les groupes de mots, les deux premières lettres de chaque mot associé constituent le code (Tableau I). Le tableur Excel a permis de calculer les différentes proportions des données de l'enquête et les coordonnées géographiques sont incorporées au logiciel ArcGIS (10.4 et 10.2) pour identifier les zones d'études et de colonisation de $P$. ruderale.

Pour le traitement des données qualitatives telles que la localité, la topographie, le type de désherbage et le type cultural, l'Analyse Factorielle de Correspondance (AFC) a été utilisé.

Cette analyse statistique est descriptive, multidimensionnelle et a pour but d'analyser le lien existant entre deux variables qualitatives (Rencher, 2002). Dans cette étude le degré d'abondance (densité) est la première variable. La localité, la topographie, le type de désherbage et le type cultural, constituent de manière consécutive la deuxième variable.

Il s'agira de vérifier si ces derniers sont susceptibles d'influencer l'abondance de l'adventice. Les liaisons sont appréciées en fonction de leur rapprochement par rapport aux axes (F1 et F2). Cette analyse a été réalisée avec le logiciel XLSat 2014.

Tableau I : Codes et classes des variables retenues dans l'étude

\begin{tabular}{llll}
\hline Variables & numéros & Classes (états) & Codes \\
\hline Localité & $\mathbf{1}$ & Adzopé & ADZOPE \\
& $\mathbf{2}$ & Bondoukou & BONDOU \\
& $\mathbf{3}$ & Daloa & DALOA \\
& $\mathbf{4}$ & Korhogo & KORHOR \\
& $\mathbf{5}$ & Man & MAN
\end{tabular}




\begin{tabular}{lcll} 
& $\mathbf{6}$ & Yamoussoukro & YAMOUS \\
& & & \\
\hline Types de cultures & $\mathbf{1}$ & Igname (et Patate douce) & IGNAME \\
& $\mathbf{2}$ & Manioc & MANIOC \\
& $\mathbf{3}$ & Banane plantain & BANPLA \\
& $\mathbf{4}$ & $\begin{array}{l}\text { Céréales (Riz, Maïs) } \\
\text { Maraîchers (Tomate, Piment, }\end{array}$ & CEREAL \\
& $\mathbf{5}$ & $\begin{array}{l}\text { Aubergine, Gombo, Chou) } \\
\text { Cultures pérennes (Cacao, }\end{array}$ & CULPEN \\
& $\mathbf{6}$ & $\begin{array}{l}\text { Café, Hévéa, Palmier à huile, } \\
\text { Anacarde) }\end{array}$ & JACHER \\
& $\mathbf{7}$ & Jachères & Manuel seul \\
& $\mathbf{1}$ & $\begin{array}{l}\text { Manuel +chimique } \\
\text { Manuel + mécanique (attelé) } \\
\text { + chimique }\end{array}$ & MANCHEC \\
\hline Type & $\mathbf{2}$ & & \\
\hline
\end{tabular}

L'analyse de variance à un facteur (ANOVA 1) a été employée pour distinguer les différentes zones d'abondances selon les sites d'études. Ce test paramétrique intervient pour comparer les moyennes des données quantitatives lorsqu'il y a plus de deux séries pour la même variable. Le degré de liberté est calculé par la formule, $\mathrm{dl}=\mathrm{n}-\mathrm{k}$ ( $\mathrm{n}$ et $\mathrm{k}$ sont respectivement les nombres d'observations et de groupes). La plus petite différence significative entre les moyennes a été fixée à 5\% (Vessereau, 1992). Lorsque la différence est significative, le test de Tukey est réalisé pour classer et connaitre les différents groupes homogènes. Les traitements appartenant à un même groupe sont affectés de la même lettre.

\section{Evaluation des paramètres \\ La densité}

Elle permet de quantifier le degré de l'enherbement. C'est le nombre d'individus par unité de surface (individus $/ \mathrm{m}^{2}$ ). Ce paramètre fut choisi car l'adventice n'est pas une espèce rhizomateuse ni stolonifère donc le dénombrement des individus est plus simple et plus précis. Les notes attribuées et leurs significations sont présentées dans le tableau II selon Barralis (1976). La formule est la suivante:

$$
\mathbf{D}=\mathbf{N} / \mathbf{S}
$$

Où $\mathrm{D}$ est la densité et $\mathrm{N}$ est le nombre d'individus de $P$. ruderale observé sur la surface $\mathrm{S}$.

Tableau II: Indice d'abondance de l'espèce $P$. ruderale selon Barralis (1976)

\begin{tabular}{l|l}
\hline Indices & Densité (individus $\left./ \mathrm{m}^{2}\right)$ \\
\hline $\mathbf{1}$ & absence \\
\hline
\end{tabular}




\begin{tabular}{l|l}
\hline $\mathbf{2}$ & 1 à 2 individus $/ \mathrm{m}^{2}$ \\
$\mathbf{3}$ & 3 à 20 individus $/ \mathrm{m}^{2}$ \\
$\mathbf{4}$ & 21 à 50 individus $/ \mathrm{m}^{2}$ \\
$\mathbf{5}$ & plus de 50 individus $/ \mathrm{m}^{2}$ \\
\hline
\end{tabular}

\section{Fréquence relative $(\mathrm{Fr})$}

La fréquence relative ( $\mathrm{Fr}$ ) d'une espèce végétale donnée se définit comme le rapport de sa fréquence absolue $(\mathrm{Fa})$ sur le nombre total $(\mathrm{N})$ de relevés effectués (Bernard, 2008).

Sa formule mathématique est la suivante:

$$
\mathbf{F r}=\mathbf{F a} / \mathbf{N}
$$

\section{Fréquence centésimale $(\mathrm{Fc})$}

C'est l'expression de la fréquence relative sous forme de pourcentage. La formule est la suivante:

$$
\mathrm{Fc}=\mathrm{Fa} / \mathrm{N} \times 100
$$

$\mathrm{Fc}=$ fréquence centésimale, $\mathrm{Fa}=$ fréquence relative de l'espèce, $P$. ruderale, $\mathrm{N}=$ nombre total $(\mathrm{N})$ de relevés effectués.

\section{Résultats \\ Enquêtes}

Le nombre de parcelles agricoles échantillonnées est de 328 et celui des enquêtés s'élève à 291 producteurs car parmi ces derniers d'autres possèdent plusieurs parcelles à différents endroits. Les résultats des enquêtes ont révélé que les agriculteurs ont identifié à plus de $74 \%$ P. ruderale comme une mauvaise herbe poussant dans leur champ, au bord des pistes, mais elle est inconnue pour certains (16\%). Ils ont indiqué une récente apparition de cette adventice, la situant à la période de 2010 à 2015 (49\%) et 42\% restent sans avis. D'autres affirment l'avoir observée durant la période 2005 à 2010 $(6 \%)$ et $1 \%$ avant l'an 2000 . L'abondance de l'adventice est très remarquable en saison des pluies ( $45 \%$ ), en outre $31 \%$ ont mentionné qu'elle est abondante aussi bien en saison sèche qu'en saison pluvieuse. Le manioc $(55 \%)$ est la principale culture rencontrée dans toutes les zones visitées sauf à Korhogo où la culture dominante est le maïs. Le désherbage manuel est pratiqué à plus de $55 \%$ avec des outils comme la machette et la daba. Le taux des producteurs utilisant le désherbage manuel associé aux méthodes chimiques est de $43 \%$. Le tableau III présente les résultats des enquêtes. L'usage de $P$. ruderale comme plante médicinale est inconnue des producteurs. 
Tableau III: Résultats des enquêtes selon les sites d'études

\begin{tabular}{|c|c|c|c|c|c|c|c|}
\hline & \multicolumn{7}{|c|}{ Chef-lieu de région } \\
\hline & Adzopé & Bondou & Daloa & Korhor & Man & Yamous & total \\
\hline $\begin{array}{ll}\text { Nombres } & \text { de } \\
\text { parcelles } & \end{array}$ & 58 & 55 & 55 & 53 & 54 & 53 & 328 \\
\hline $\begin{array}{l}\text { Nombre } \\
\text { d'enquêtés }\end{array}$ & 58 & 49 & 40 & 49 & 46 & 49 & 291 \\
\hline $\begin{array}{l}\text { Identification de } \\
P . \text { ruderale }(\%) \\
1=\text { oui } ; 2=\text { non } \\
3=\text { inconnue }\end{array}$ & $1=100$ & $\begin{array}{l}1=57,14 \\
2=42,86\end{array}$ & $\begin{array}{l}1=97,5 \\
2=2,5\end{array}$ & $3=100$ & $\begin{array}{l}1=97,83 \\
2=2,17\end{array}$ & $\begin{array}{l}1=95,92 \\
2=4,08\end{array}$ & $\begin{array}{l}1=74,57 \\
2=8,59 \\
3=16,84\end{array}$ \\
\hline $\begin{array}{l}\text { Période } \\
\text { d'observation } \\
1=\text { avant } 2000 \\
2=2005-2010 \\
3=2010-2015 \\
4=\text { sans avis }\end{array}$ & $\begin{array}{l}1=0 \\
2=6,9 \\
3=82,75 \\
4=10,35\end{array}$ & $\begin{array}{l}1=0 \\
2=2,05 \\
3=28,57 \\
4=69,38\end{array}$ & $\begin{array}{l}1=0 \\
2=0 \\
3=75 \\
4=25\end{array}$ & $\begin{array}{l}1=0 \\
2=0 \\
3=0 \\
4=100\end{array}$ & $\begin{array}{l}1=0 \\
2=8,7 \\
3=56,52 \\
4=34,78\end{array}$ & $\begin{array}{l}1=8,18 \\
2=22,44 \\
3=53,06 \\
4=16,32\end{array}$ & $\begin{array}{l}1=1,37 \\
2=6,88 \\
3=49,49 \\
4=42,26\end{array}$ \\
\hline $\begin{array}{l}\text { Période } \\
\text { d'abondance }(\%) \\
1=\text { pluie } \\
2=\text { pluie+sèche } \\
3=\text { sans avis }\end{array}$ & $\begin{array}{l}1=62,07 \\
2=37,93\end{array}$ & $\begin{array}{l}1=61,22 \\
3=38,78\end{array}$ & $\begin{array}{l}1=65 \\
2=35\end{array}$ & $3=100$ & $\begin{array}{l}1=41,31 \\
2=58,69\end{array}$ & $\begin{array}{l}1=40,82 \\
2=59,18\end{array}$ & $\begin{array}{l}1=45,01 \\
2=31,61 \\
3=23,38\end{array}$ \\
\hline $\begin{array}{l}\text { Principales } \\
\text { cultures }(\%)\end{array}$ & $\begin{array}{l}\text { Manioc } \\
(85)\end{array}$ & $\begin{array}{l}\text { Manioc } \\
(70) \\
\text { Igname et } \\
\text { Anacarde } \\
(12,5) \\
\end{array}$ & $\begin{array}{l}\text { Manioc } \\
(42) \\
\text { Igname } \\
(25) \\
\text { Maïs (15) }\end{array}$ & $\begin{array}{l}\text { maïs } 60,37 \\
\text { Arachide } \\
13,2\end{array}$ & $\begin{array}{l}\text { Manioc } \\
(46 \\
\text { Riz (22), }\end{array}$ & $\begin{array}{l}\text { Manioc (38) } \\
\text { Igname (21) } \\
\text { Tomate (11) }\end{array}$ & $\begin{array}{l}\text { Manioc } \\
55,32\end{array}$ \\
\hline $1=\mathrm{MANSEU}$ & $1=77,59$ & $1=56,86$ & $1=56,6$ & $1=13,20$ & $1=79,63$ & $1=49,06$ & $1=55,86$ \\
\hline $2=\mathrm{MANCHI}$ & $2=20,69$ & $2=43,14$ & $2=43,64$ & $2=83,01$ & $2=20,37$ & $2=50,94$ & $2=43,20$ \\
\hline $\begin{array}{l}3=\text { MANMEC } \\
(\%)\end{array}$ & $3=1,72$ & $3=0$ & $3=0$ & $3=3,79$ & $3=0$ & $3=0$ & $3=0,94$ \\
\hline
\end{tabular}

\section{Répartition de Porophyllum ruderale en Côte d'Ivoire}

$P$. ruderale a été identifiée dans 235 exploitations agricoles sur 328, soit une fréquence centésimale de 71,64\%. Elle est présente à Adzopé, Bondoukou, Daloa, Man et Yamoussoukro mais est absente à Korhogo (Figure 2). Les fréquences centésimales (Fc) indiquent un taux de $100 \%$ pour Adzopé et Daloa, il est de 92,59\% à Man, 90,57\% à Yamoussoukro, 43,63\% à Bondoukou et $0 \%$ à Korhogo selon les villages visités (Tableau IV). En dehors de nos sites d'études, l'adventice a été observée dans d'autres localités notamment à San-Pedro, Abidjan, Divo, Issia, Soubré; Gagnoa, Bouaké, Katiola; Duekoué, Biankouman; Bouna, Ferkessédougou, M'bahiakro, Bouaflé, Zuénoula, Sassandra, Toumodi-Sakassou. 


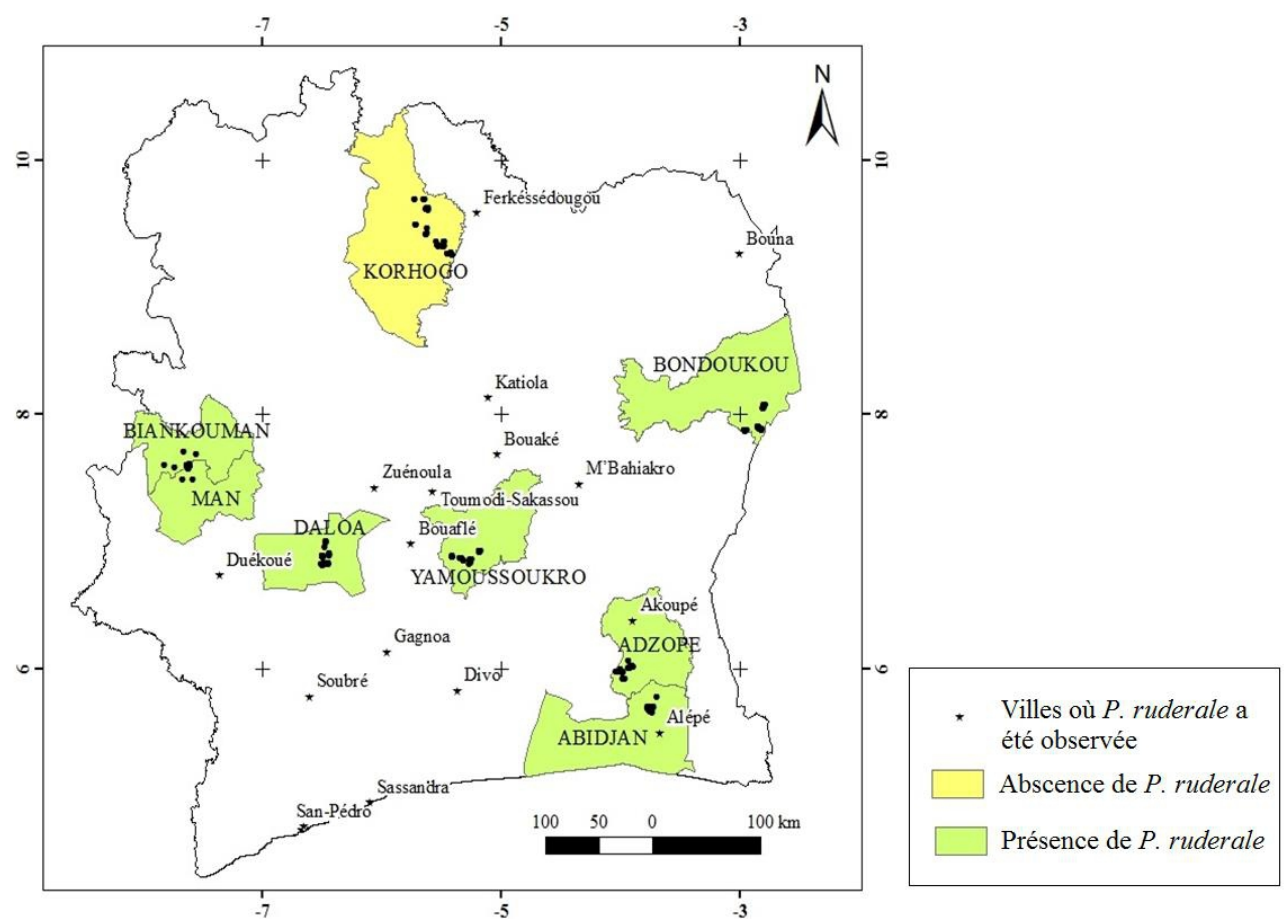

Figure 2:Répartition de Porophyllum ruderale en fonction des zones d'études et visitées (source, couche vectorielle, openStreetMap, modifiée par KPLA F, 2017)

Le degré d'infestation de $P$. ruderale (densité) est significativement différent d'une localité à une autre $(F=112,98 ; P<0,0001)$. Le test de Tukey a permis de distinguer quatre classes (Tableau IV). Il s'agit de la classe "a" composée de la localité d'Adzopé, avec une densité comprise entre 3 à 50 individus $/ \mathrm{m}^{2}$; de "b" regroupant les localités de Daloa, Yamoussoukro et Man, pour une densité de 1 à 20 individus $/ \mathrm{m}^{2}$; de "ce" comprenant la localité de Bondoukou avec une faible densité, 1 à 2 individus $/ \mathrm{m}^{2}$ et de "d" représentée par la localité de Korhogo avec une absence d'individus de $P$. ruderale.

Tableau IV : Densité et fréquence estimées de $P$. ruderale en fonction des zones d'études

Densité de $P$. ruderale Fréquences

centésimales $(\%)$

Localités

ADZOPE

DALOA

YAMOUSOUKRO

MAN

BONDOUKOU

KORHOGO

$d d l$
Moyennes \pm Ecarts types

$3,29 \pm 0,61 \mathbf{a}$

100

$2,30 \pm 0,10 \mathbf{b}$

100

$2,30 \pm 0,09 \mathbf{b}$

90,57

$2,05 \pm 0,02 \mathbf{b}$

92,59

$1,56 \pm 0,27$ c

43,63

$1 \pm 0,54 \mathbf{d}$

0

5

Total 


\begin{tabular}{lll}
\hline$F$ & 112,98 & 71,64 \\
$p$ & $<0,0001$ & \\
\hline$d d l:$ degré de liberté $F \cdot$ valeur du rapport des variances, $p:$ valeur de la probabilité, a, b, c, d
\end{tabular}
: moyennes suivies des mêmes lettres ne sont pas significativement différentes au seuil $\alpha=$ 0,05 (test de Tukey).

L'observation d'un individu adulte de $P$. ruderale dans une zone suppose qu'elle y a trouvé des conditions environnementales favorables à son développement. Les températures des zones de présence de $P$. ruderale varient de 21 à $28^{\circ} \mathrm{C}$ et la pluviométrie s'élève de 1100 à $2400 \mathrm{~mm} / \mathrm{an}$. L'abondance de $P$. ruderale observée selon les sites d'études est importante dans les zones forestières donc par extrapolation et selon le climat, la carte de la distribution de $P$. ruderale est réalisée à la figure 3.

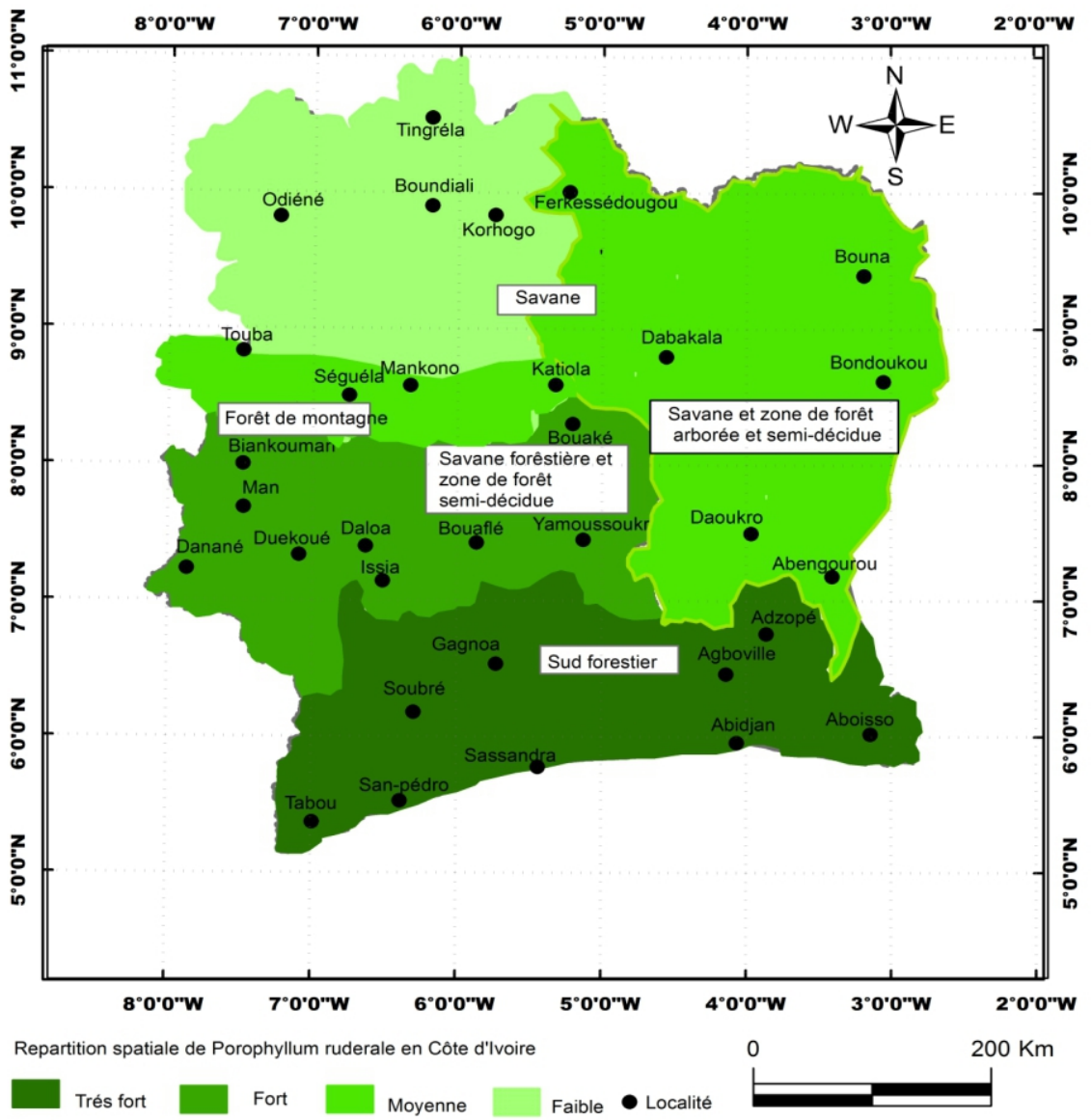

Abondance de $P$. ruderale

Source: Couche vectorielle, OpenStreetMap, modifiée par KPLA F, 2017

Figure 3: Répartition selon l'abondance de $P$. ruderale en Côte d'Ivoire. 


\section{Répartition de la densité de $\boldsymbol{P}$. ruderale en fonction des « localités »}

La localité, par sa position géographique, permet de décrire les caractéristiques écologiques (température, pluviométrie et type de végétation) du milieu. Elle est donc susceptible d'expliquer la présence ou l'absence d'une espèce. L'AFC effectuée pour l'analyse de l'abondance de $P$. ruderale selon les localités différencie quatre groupes (Figure 4). Le premier est celui de la localité d'Adzopé où, l'adventice y est très abondante, le deuxième comprend les localités de Daloa, Man et Yamoussoukro où l'abondance est relativement forte. Le troisième comprend la localité de Bondoukou avec une faible abondance, enfin le dernier est la localité de Korhogo, l'adventice y est rare.

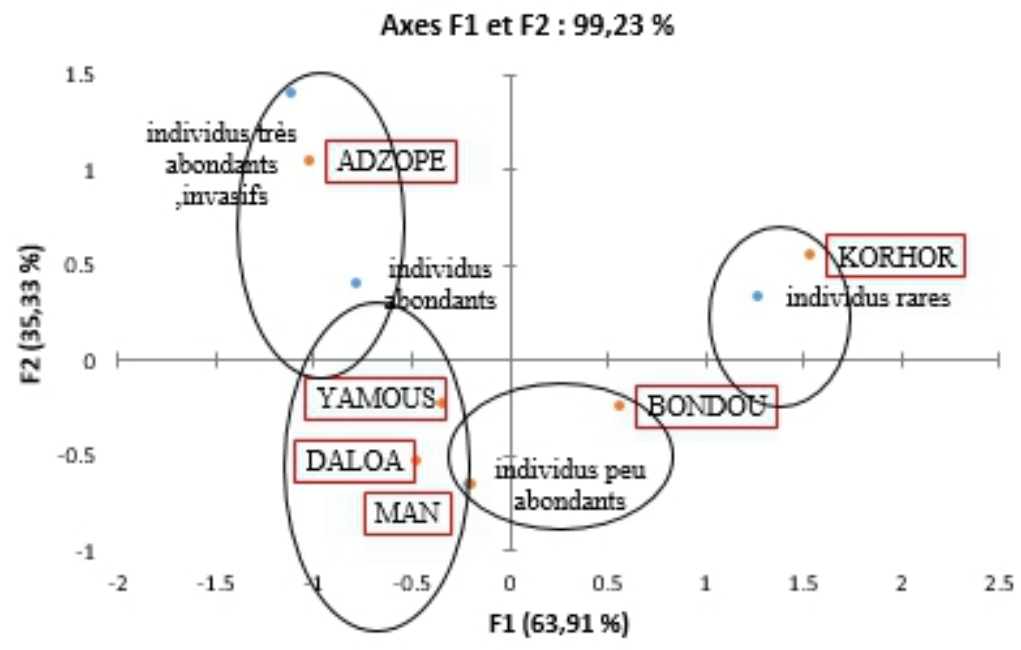

Figure 4 : Répartition de la densité de $P$. ruderale en fonction de la «localité»

\section{Répartition de la densité de P.ruderale en fonction de la « topographie »}

La topographie peut être à mesure d'expliquer la répartition d'une espèce par rapport à d'autres, en caractérisant leur zone de prédominance. L'analyse statistique (l'AFC) réalisée sur l'abondance de $P$. ruderale en fonction de la topographie distingue deux groupes (Figure 5). Le premier est composé des champs situés sur des plateaux où l'abondance de cette adventice est élevée. Le second groupe est constitué des mi-versants et des bas-fonds avec une faible abondance de l'adventice. 
(axes F1 et F2 : 100,00\%)

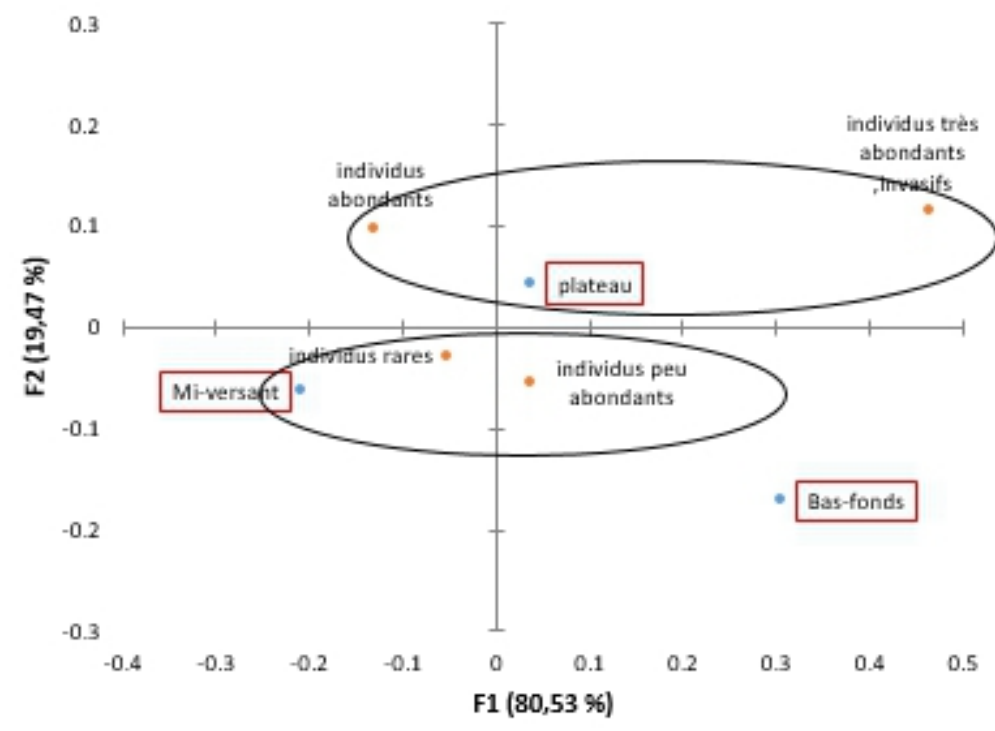

Figure 5 : Répartition de la densité de $P$. ruderale en fonction de la «topographie»

Répartition de la densité de $P$. ruderale en fonction du « type de désherbage »

Les méthodes de désherbages influencent les levées de l'adventice. L'AFC effectuée (Figure 6) pour l'analyse de l'abondance de $P$. ruderale selon les méthodes de désherbage détermine deux groupes. Celui des techniques manuel seul et manuel +chimique, où les parcelles présentent de fortes densités. Le second regroupe des parcelles désherbées avec la combinaison des trois méthodes (manuel + chimique + mécanique attelée) où on observe une faible abondance. 


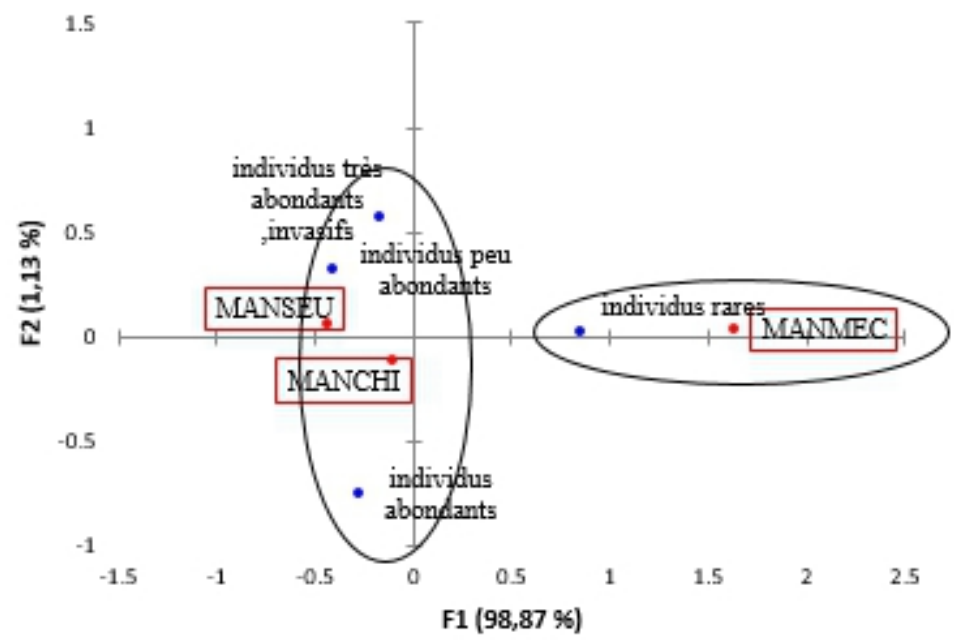

Figure 6 : Répartition de la densité de $P$. ruderale en fonction du «type de désherbage »

\section{Répartition de la densité de $\boldsymbol{P}$. ruderale en fonction de la « culture »}

Certaines cultures, par leurs architectures et leurs itinéraires techniques favorisent ou non la prolifération des adventices. Trois groupes ont été dégagés par l'AFC (Figure 7). Le premier groupe comprend les champs de manioc et de banane où l'abondance de $P$. ruderale est très importante (plus de 21 individus $/ \mathrm{m}^{2}$ ). Le second présente des champs d'ignames et des jachères moyennement infestés ( 1 à 20 individus $/ \mathrm{m}^{2}$ ). Le dernier est composé des cultures pérennes, les maraîchères et les céréalières avec très peu de représentants de $P$. ruderale (abondance faible avec 1 à 2 individus $/ \mathrm{m}^{2}$ ).

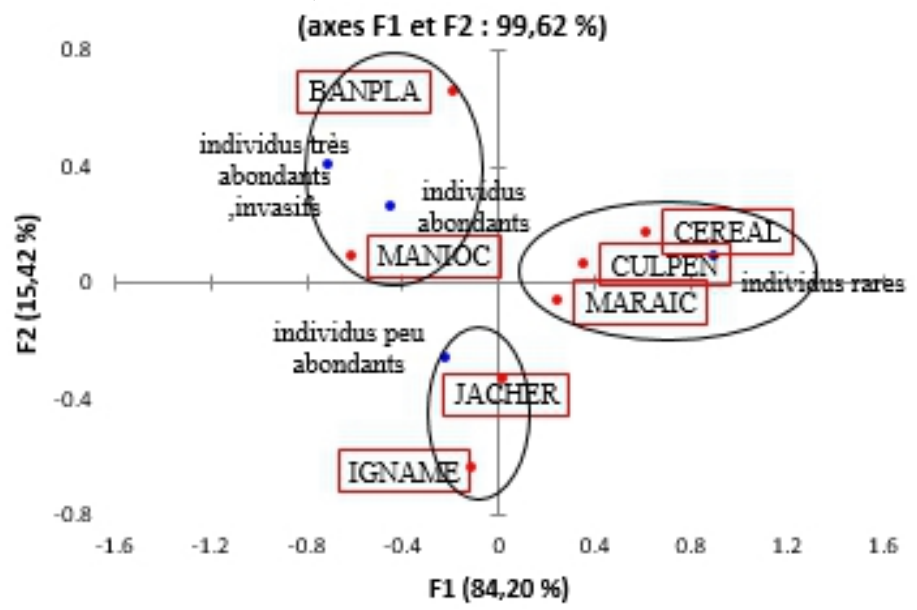

Figure 7 : Répartition de la densité de $P$. ruderale en fonction de la «culture » 


\section{Discussion}

Au cours de notre enquête, la majorité des agriculteurs ont affirmé la récente introduction de $P$. ruderale dans leurs plantations (2010-2015). Cette espèce n'est pas une plante ornementale comme Eichhornia crassipes (Pontederiaceae), la jacinthe d'eau, ni un engrais vert comme Leucaena leucocephala appartenant à la famille des Fabaceae (Aké Assi, 2002; AkéAssi \& Ipou Ipou, 2006). Elle a donc dû être introduite accidentellement en Côte d'Ivoire. Notre démarche est la même que Danho et al. (2014) qui ont qualifié d'accidentelle l'introduction de Chromolaena odorata (Asteraceae).

$P$. ruderale est présente dans tous les types de climats et de végétations du territoire ivoirien. Son aire de distribution s'étend du Sud forestier aux régions savanicoles du Nord-Est en passant par le Centre pré-forestier. Cela peut s'expliquer par le fait qu'elle produise d'importantes quantités de semences très légères avec une touffe de poils à leur extrémité (FrangiotePallone \& De Souza, 2012), facilitant ainsi leur dispersion sur de longues distances. De plus, cette espèce s'adapte à différents types de sols même pauvres ou sableux (Kissmam \& Groth, 1999), et ses semences ont un taux de germination élevé en milieu naturel, variant de 40 à $70 \%$ (Kpla et al., 2017). Toutefois, $P$. ruderale est plus abondante en zones forestières et préforestières qu'en zone Soudanienne. Cette différence pourrait être liée aux conditions climatiques de ces différentes zones. En effet, les précipitations sont plus importantes dans les zones fortement colonisées par $P$. ruderale (1100 à $2400 \mathrm{~mm} / \mathrm{an}$ ) que dans celles moins infestées. Les températures de ces zones sont également différentes, les températures sont relativement faibles en zone forestière et pré-forestière qu'en zone soudanienne. La pluviométrie et la température pourraient donc être des facteurs discriminants dans la répartition de cette espèce.

En plus de ces facteurs climatiques, les pratiques culturales peuvent expliquer la différence de degré d'infestation de $P$. ruderale observée entre le Nord et le Sud de la Côte d'Ivoire. Au Nord de la Côte d'Ivoire, les principales cultures sont de cycles courts, particulièrement le maïs et l'arachide, qui ont une durée de 2 à 4 mois. Il en est de même pour les cultures marâichères qui de plus, se pratiquent sur de petites surfaces. L'entretien de la parcelle est donc moins long et régulier par rapport à d'autres spéculations notamment le manioc (cultivé principalement en zone forestière), dont le cycle dure 9 à 24 mois. En outre, les populations du Nord appliquent les méthodes de lutte intégrée en combinant manuelle, chimique et mécanique attelée, ce qui pourrait limiter l'avancée de $P$. ruderale. Ces résultats concordent avec ceux obtenus par Kouamé (2014) en riziculture dans les régions du Centre. Celuici a montré que la mécanisation couplée à l'application d'herbicide en prélevée permet de réduire la nuisibilité des adventices. 
$P$. ruderale est principalement abondante en culture du manioc avec plus de 21 individus $/ \mathrm{m}^{2}$. Les travaux de Yapi (2017) dans la région de la Mé, au Sud de la Côte d'Ivoire, ont indiqué que cette espèce fait partie des mauvaises herbes majeures responsables des pertes de rendements en culture du manioc. Selon cet auteur, le manque de désherbage serait la cause de cette invasion. Or, la fertilité du sol et la pluviométrie élevée en ces zones forestières favorisent la croissance rapide des adventices. Le nombre insuffisant de désherbages, mais de plus effectués généralement avec les outils manuel (95 $\%$ d'usage de la machette) serait la raison de l'invasion rapide de $P$. ruderale. En effet, le fauchage à la machette active le stock semencier entraînant une germination en masse de $P$. ruderale. En outre, le taux de levée s'élève à plus de $80 \%$ lorsque les graines sont déposées à la surface du sol (Kpla et al., 2017). Le type de culture pratiqué par le paysan influence également la répartition de $P$. ruderale. Cette espèce est très répandue en culture du manioc que dans les céréales et moins dans les pérennes. L'ombrage créé par les cultures pérennes pourrait expliquer la rareté de $P$. ruderale en leur sein. En effet, les travaux de Kpla et al. (2017) sur $P$. ruderale ont montré un taux de levée faible (10\%) en milieu sous ombrage par rapport au milieu ouvert (40-70\%).

$P$. ruderale colonise tous les profils topographiques (plateaux, miversants et bas-fonds) mais sa densité est plus élevée sur les plateaux que sur les autres types.

\section{Conclusion}

Porophyllum ruderale Jacq. Cass est une adventice nouvellement observée en Côte d'Ivoire. Cette Asteraceae produit beaucoup de semences et est une plante envahissante en Amérique latine. La présente étude a permis d'identifier les zones de colonisation et l'abondance de cette adventice. Les agriculteurs soutiennent sa récente introduction durant la période 2010 à 2015 (environ 50\%). Cette espèce est représentée dans toutes les régions avec une fréquence de 71,64\% et une abondance variable. Celle-ci est élevée dans les zones forestières particulièrement au Sud, au Centre et à l'Ouest, mais est faible dans les zones de l'Est et du Nord. Les milieux agroécologiques de colonisation de l'adventice indiquent une variation de température de 21 à 28 ${ }^{\circ} \mathrm{C}$ et une pluviométrie de 1100 à $2400 \mathrm{~mm} / \mathrm{an}$. Porophyllum ruderale se développe sur tous les types topographiques (plateaux, mi-versants et basfonds), mais est abondante dans les plateaux. La densité de la mauvaise herbe est plus importante dans la culture du manioc (plus de 21 individus $/ \mathrm{m}^{2}$ ). La dispersion de Porophyllum ruderale est remarquable et il serait pratiquement impossible de la stopper surtout que ses semences sont très volatiles. Néanmoins, certaines pratiques pourraient freiner cette diffusion dans les agrosystèmes, notamment éliminer les individus de l'adventice avant la 
floraison. La technique du faux semis et la pratique des labours profonds pourront être préconisées.

\section{References:}

1. Aké-Assi, E. (2002). Contribution à l'étude des plantes ornementales cultivées dans la région d'Abidjan et de San-Pédro en Côte d'Ivoire. Thèse, Université de Cocody, Abidjan, 242 p.

2. Aké-Assi, E., \& Ipou Ipou, J. (2006). Litsea glutinosa (Lour.) C. B. Robinson (Lauraceae): de son introduction en Côte d'Ivoire à la conquête de l'espace. Sci. Nat. 3 (2): 113-220.

3. Bayer, A. G. (1992). Important Crops of the World and their Weeds (Scientific and Common Names, Synonyms and W.S.S.A. /W.S.S.J. Approved Computer Codes). Second ed., Bayer ed., Leverkussen, Federal Republic of Germany ;1682 p.

4. Bakayoko, S., Soro, D., Kouadio, K. K. H., Konan-Waidhet, A. B., Angui, P. (2014). "Characteristics of Tonkpi Mountain soils and plateaus soils in West Côted'Ivoire » [archive], sur .heraldjournals.org (consulté le 22 juin 2014).

5. Barralis, G. (1976). Méthode d'étude des groupements adventices des cultures annuelles, application à la Côte d'or. $V$ ème colloque international sur l'écologie et la Biologie des Mauvaises herbes (Dijon) I, 59-68.

6. BNETD/CCT. (2007). Côte d'Ivoire, « Etat de la production cartographique et de la gestion des informations géographiques de référence », Abidjan, $32 \mathrm{p}$.

7. Bernard, B. (2008). Le nouveau contexte du développement de l'agriculture dans le monde. Dossier, Edition FARM, 28 p.

8. Coulibaly, T., Akpesse, A. A. M., Yapi, A., Zirihi, G. N., \& Kouassi, K. P. (2014). Dégâts des termites dans les pépinières de manguiers du nord de la Côte d'Ivoire (Korhogo) et essai de lutte par utilisation d'extraits aqueux de plantes. Journal of Animal \& Plant Sciences, Vol.22, Issue 3: 3455-3468.

9. Danho, F. R. N., Djah, F. M., Koné, M., \& Kouadio, Y. L. (2014). Inventaire préliminaire des plantes envahissantes de la Côte d'Ivoire, Journal of Animal \&Plant Sciences, Vol.22, Issue 2: 3439-3445

10. De Marinis, G., Lepos, A., Friebolin, L. P., \& Ram, M. (1980). Capacité de reproduction de Porophyllum ruderale (Jacq.) Cass., Institut de Biosciences, University Cet -double Paulista $<<$ Julio Mesquita Filho de>>, science et des Langues de São José do Rio Preto, Plant mauvaises herbes III (1):55-57

11. Fonseca, M. C. M., Meira, R. M. S. A., \& Casali, M. D. V. (2006). Anatomie d'organes végétatifs et histolocalisation des lipides et des 
composés phénoliques dans Porophyllum ruderale (Asteraceae). Planta Daninha.; Vol.24, no.4, p. 707-13.

12. Frangiote-Pallone, S., \& De Souza, L. A. (2012). Ontogenia del papus y Cipsela en Asteraceae: las consideracionese structurales de la Catégorie tribale, Departamento de Biologia, Universidad e Estadual de Maringá, Brésil, Avenida Colombo 5790, (87020 à 900).

13. Frangiote-Pallone, S., \& De Souza, L. A. (2014). Pappus and cypsel aontogeny in Asteraceae: structural considerations of the tribal category, Revista Mexicana de Biodiversidad, Vol 85, Issue 1, Pages 62-77.

14. Johnson, D. E. (1997). Les adventices en riziculture en Afrique de l'Ouest. ADRAO, Imprint Design. United Kingdom. 312 p.

15. Kissmam, K. G., \& Groth, D. (1999). Plantas infestantes e nocivas. Tomo II. 2. ed. São Paulo: Basf,.420 p. ;414-417 e 392-395.

16. Kouamé, K. F. (2014). Contraintes liées à la production rizicole, étude de la flore et de la végétation adventices des rizicultures dans la région du Bélier (Centre de la Côte d' Ivoire). Thèse de l'Université Félix Houphouët Boigny, Abidjan, 145 p.

17. Kpla, C. F., Touré, A., \& Ipou Ipou, J. (2017). Germination Capacity of Porophyllum ruderale (Jacq.) Cass (Asteraceae) A Food Crop Weed in Côte d'Ivoire ; Journal of Agriculture and Environmental Sciences, Vol. 6, No. 2, pp. 114-120.

18. Krogba, Y. N., Kouakou, Y. K. N., Gohi, B. Z. F., Rusu, E., \& YaoKouamé, A. (2016). Distribution et comportement des éléments traces métalliques dans les cambisols manganesiferes des sites volcanosedimentaires de Côte d'Ivoire, Lucrările Seminarului Geografic "Dimitrie Cantemir" Nr. 43

19. Le Bourgeois, T. (1993). Les mauvaises herbes dans la rotation cotonnière au Nord Cameroun (Afrique). Thèse de Doctorat, Université de Montpellier II Sciences et Techniques du Languedoc, Montpellier, France, 249 p.

20. Rencher, C. A. (2002). Methods of Multivariate Analysis. A. John Wiley \& Sons, INC. Publication, New York (USA), 708 p.

21. Rodenburg, J., \& Johnson, D. E. (2009).Weed management in ricebased cropping systems in Africa. Advances in Agronomy, 103:149218.

22. Tra Bi, Z. A., Brou, Y. T \& Mahé, G. (2015). Analyse par télédétection des conditions bioclimatiques de végétation dans la zone de contact forêt-savane de Côte d'Ivoire : cas du «V» Baoulé. XXVIIIe Colloque de l'Association Internationale de Climatologie, Liège.

23. Vessereau, A. (1992). Méthodes statistiques en biologie et en agronomie Paris: Diffusion TEC \& DOC Lavoisier, 337 p. 
24. Yao, A. C., Ipou Ipou, J., Edson, L. B., Djédoux, M. A., \& Koné, W. M. (2017). Caractérisation physiologique et évaluation du comportement germinatif de semences de Rottboellia cochinchinensis, Euphorbia heterophylla, et Porophyllum ruderale, trois adventices des rizières de Côte d'Ivoire, UFR Sciences de la Nature/Centre Suisse de Recherches Scientifiques en Côte d'Ivoire (CSRS), European Scientific Journal, édition vol.13, No.3 ISSN: 1857 - 7881(Print) e ISSN 1857- 7431

25. Yapi, A-F. (2017). Mauvaises herbes majeures et itinéraires techniques de désherbage des cultures vivrières de la région de la Mé, au sud-est de la Côte d'Ivoire : cas du bananier plantain et du manioc, Thèse de l'Université Félix Houphouët Boigny, Abidjan, Côte d'Ivoire, 179 p.

26. Youan, T. M., Lasm, T., Jourda, J. P., Kouamé, K. F., \& Razack, M. (2008). Cartographie structurale par imagerie satellitaire ETM+ de Landsat-7 et analyse des réseaux de fractures du socle précambrien de la région de Bondoukou (Nord-est de la Côte d'Ivoire) (Sous presse) Dans la revue Télédétection. 